PROCEEDINGS OF THE

AMERICAN MATHEMATICAL SOCIETY

Volume 127, Number 1, January 1999, Pages 155-165

S 0002-9939(99)04763-2

\title{
GLOBAL BIFURCATION IN GENERIC SYSTEMS OF NONLINEAR STURM-LIOUVILLE PROBLEMS
}

\author{
BRYAN P. RYNNE
}

(Communicated by Hal L. Smith)

\begin{abstract}
We consider the system of coupled nonlinear Sturm-Liouville boundary value problems

$$
\begin{gathered}
L_{1} u:=-\left(p_{1} u^{\prime}\right)^{\prime}+q_{1} u=\mu u+u f(\cdot, u, v), \quad \text { in }(0,1), \\
a_{10} u(0)+b_{10} u^{\prime}(0)=0, \quad a_{11} u(1)+b_{11} u^{\prime}(1)=0, \\
L_{2} v:=-\left(p_{2} v^{\prime}\right)^{\prime}+q_{2} v=\nu v+v g(\cdot, u, v), \quad \text { in }(0,1), \\
a_{20} v(0)+b_{20} v^{\prime}(0)=0, \quad a_{21} v(1)+b_{21} v^{\prime}(1)=0,
\end{gathered}
$$

where $\mu, \nu$ are real spectral parameters. It will be shown that if the functions $f$ and $g$ are 'generic' then for all integers $m, n \geq 0$, there are smooth 2-dimensional manifolds $\mathcal{S}_{m}^{1}, \mathcal{S}_{n}^{2}$, of 'semi-trivial' solutions of the system which bifurcate from the eigenvalues $\mu_{m}, \nu_{n}$, of $L_{1}, L_{2}$, respectively. Furthermore, there are smooth curves $\mathcal{B}_{m n}^{1} \subset \mathcal{S}_{m}^{1}, \mathcal{B}_{m n}^{2} \subset \mathcal{S}_{n}^{2}$, along which secondary bifurcations take place, giving rise to smooth, 2-dimensional manifolds of 'nontrivial' solutions. It is shown that there is a single such manifold, $\mathcal{N}_{m n}$, which 'links' the curves $\mathcal{B}_{m n}^{1}, \mathcal{B}_{m n}^{2}$. Nodal properties of solutions on $\mathcal{N}_{m n}$ and global properties of $\mathcal{N}_{m n}$ are also discussed.
\end{abstract}

\section{INTRODUCTION}

We consider the system of coupled nonlinear Sturm-Liouville boundary value problems

$$
\begin{gathered}
L_{1} u:=-\left(p_{1} u^{\prime}\right)^{\prime}+q_{1} u=\mu u+u f(\cdot, u, v), \quad \text { in }(0,1), \\
a_{10} u(0)+b_{10} u^{\prime}(0)=0, \quad a_{11} u(1)+b_{11} u^{\prime}(1)=0, \\
L_{2} v:=-\left(p_{2} v^{\prime}\right)^{\prime}+q_{2} v=\nu v+v g(\cdot, u, v), \quad \text { in }(0,1), \\
a_{20} v(0)+b_{20} v^{\prime}(0)=0, \quad a_{21} v(1)+b_{21} v^{\prime}(1)=0,
\end{gathered}
$$

where: $a_{i j}, b_{i j}$ are real numbers with $\left|a_{i j}\right|+\left|b_{i j}\right|>0, i=1,2, j=0,1 ; \mu, \nu$ are real parameters; $p_{i}, q_{i}, i=1,2$, are real-valued, continuous functions on $[0,1]$, with $p_{i}$ continuously differentiable and positive; the functions $f, g:[0,1] \times \mathbb{R}^{2} \rightarrow \mathbb{R}$ are $C^{k}, k \geq 3$, and $f(x, 0,0)=g(x, 0,0)=0, x \in[0,1]$.

For $i=1,2$, let

$$
\begin{aligned}
& Y_{i}=\left\{w \in C^{1}([0,1]): w \text { satisfies the boundary conditions in }(1 . i)\right\}, \\
& X_{i}=Y_{i} \cap C^{2}([0,1]), \quad X_{i}^{0}=X_{i} \backslash\{0\},
\end{aligned}
$$

Received by the editors May 2, 1997.

1991 Mathematics Subject Classification. Primary 34B15; Secondary 34B24, 58E07.

Key words and phrases. Global bifurcation, genericity, Sturm-Liouville systems. 
and let $Z=C^{0}([0,1])$; these spaces are endowed with their usual sup-norms, which will be denoted by $\|\cdot\|_{r}, r \geq 0$ (see [9] or [15]). Let $E$ be the product space $\mathbb{R} \times X_{1} \times \mathbb{R} \times X_{2}$ together with the norm $\|(\mu, u, \nu, v)\|^{2}=|\mu|^{2}+\|u\|_{2}^{2}+|\nu|^{2}+\|v\|_{2}^{2}$ (similar norms will be used on other product spaces introduced below). A solution of (1)-(2) is an element $(\mu, u, \nu, v)$ of $E$ which satisfies these equations. Clearly, $(\mu, u, \nu, v)=(\mu, 0, \nu, 0)$ is a solution of the system (1)-(2) for all $(\mu, \nu) \in \mathbb{R}^{2}$; we will call such solutions trivial. Any solution of (1)-(2) having the form $(\mu, u, \nu, 0)$, $u \neq 0$, or $(\mu, 0, \nu, v), v \neq 0$, will be called semi-trivial, while any solution having the form $(\mu, u, \nu, v), u \neq 0, v \neq 0$, will be called non-trivial.

Putting $v=0$ in (1) yields the problem

$$
L_{1} u=\mu u+u f(\cdot, u, 0), \quad u \in X_{1},
$$

where $f(\cdot, u, 0)$ now denotes the element of $Z$ defined by $x \rightarrow f(x, u(x), 0)$ and the other terms in (3) have a natural interpretation in the setting of the above Banach spaces. The results of [11] can be applied to this problem. Let $\mu_{m}^{0}, m=$ $0,1, \ldots$ (respectively, $\nu_{n}^{0}, n=0,1, \ldots$ ), denote the strictly increasing sequence of eigenvalues of $L_{1}$ (respectively, $L_{2}$ ). Then it is shown in [11] that for each $m \geq 0$ there exists an unbounded continuum $\mathcal{C}_{m}^{1}$ (in the topology of $\mathbb{R} \times Y_{1}$ ) of solutions $(\mu, u)$ of $(3)$ which bifurcates from $\left(\mu_{m}^{0}, 0\right)$. Any solution $(\mu, u) \neq\left(\mu_{m}^{0}, 0\right)$ in $\mathcal{C}_{m}^{1}$ has $u \neq 0$, and $u$ has exactly $m$ simple zeros in $(0,1)\left(u^{\prime} \neq 0\right.$ at a simple zero - see [11] for more details). Similarly, putting $u=0$ in (2) yields

$$
L_{2} v=\nu v+v g(\cdot, 0, v), \quad v \in X_{2},
$$

and for each $n \geq 0$ a continuum $\mathcal{C}_{n}^{2}$ (in $\mathbb{R} \times Y_{2}$ ) of solutions of (4) bifurcates from $\left(\nu_{n}^{0}, 0\right)$ - these continua have similar properties to those of the continua $\mathcal{C}_{m}^{1}$.

Clearly, for each $m, n \geq 0$ the sets

$$
\mathcal{S}_{m}^{1}=\mathcal{C}_{m}^{1} \times \mathbb{R} \times\{0\}, \quad \mathcal{S}_{n}^{2}=\mathbb{R} \times\{0\} \times \mathcal{C}_{n}^{2}
$$

are continua of semi-trivial solutions of (1)-(2) (except at points of the form $\left(\mu_{m}^{0}, 0, \nu, 0\right)$ and $\left(\mu, 0, \nu_{n}^{0}, 0\right)$ respectively). It is shown in the papers [2], [3], [4], [5], [6] that at certain points on $\mathcal{S}_{m}^{1}, \mathcal{S}_{n}^{2}$, secondary bifurcation may take place whereby continua of non-trivial solutions bifurcate from these continua of semi-trivial solutions. This is clearly a complicated process, and the cited papers attempt to understand the structure of the resulting continua of non-trivial solutions, and the way they join to $\mathcal{S}_{m}^{1}, \mathcal{S}_{n}^{2}$. In particular, one desires to know if pairs of continua $\mathcal{S}_{m}^{1}, \mathcal{S}_{n}^{2}$ are 'linked' by these bifurcating continua. Under certain conditions on $f$ and $g$ it is shown in [4] that such linking occurs. It is also shown that the linking continua have topological dimension $\geq 2$.

In this paper it will be shown that if the functions $f$ and $g$ are 'generic' (this will be made precise below), then for each $m$ and $n$ the continua $\mathcal{C}_{m}^{1}, \mathcal{C}_{n}^{2}$ are smooth curves, so the continua $\mathcal{S}_{m}^{1}, \mathcal{S}_{n}^{2}$ are smooth 2-dimensional manifolds, and secondary bifurcations take place along smooth curves $\mathcal{B}_{m n}^{1} \subset \mathcal{S}_{m}^{1}, \mathcal{B}_{m n}^{2} \subset \mathcal{S}_{n}^{2}$, giving rise to a smooth, 2-dimensional manifold of solutions, $\mathcal{N}_{m n}$, containing both the curves $\mathcal{B}_{m n}^{1}, \mathcal{B}_{m n}^{2}$, which is such that any solution in $\mathcal{N}_{m n}$ which does not lie on these curves is non-trivial. In addition, the non-trivial solutions lying on the manifold $\mathcal{N}_{m n}$ have nodal properties related to the integers $m, n$. Global properties of these manifolds are also discussed.

We conclude this introductory section with some definitions and notation concerning transversality and genericity which will be used below (see [14] or Section 
73.13 of [16] for further details). Let $B_{1}, B_{2}$, be Banach spaces and $U \subset B_{1}$ be an open set. A $C^{k}$ mapping $H: U \rightarrow B_{2}$ is Fredholm if the derivative $D H(b): B_{1} \rightarrow B_{2}$ is a (linear) Fredholm operator for all $b \in U$; we say that $H$ is transverse (to 0 ) if it is Fredholm and, at all points $b^{0} \in H^{-1}(0), D H\left(b^{0}\right)$ is surjective. A subset of a topological space is said to be residual if it contains the intersection of a countable collection of open dense sets. Note that the intersection of a countable collection of residual sets is also residual. A property of the elements of a topological space is said to be generic if it holds for all elements in a residual subset of the space.

Since we wish to discuss properties of the system (1)-(2) which hold for 'generic' functions $f$ and $g$, we need an appropriate space of functions and a topology on this space. Let $\mathcal{F}$ be the set of all real valued $C^{k}$ functions defined on $[0,1] \times \mathbb{R}^{2}$. We define a topology on $\mathcal{F}$ as follows. For any $f \in \mathcal{F}$ and any continuous, positive function $\epsilon: \mathbb{R}^{2} \rightarrow \mathbb{R}_{+}$, we define an $\epsilon$-neighbourhood of $f$ by

$$
\begin{aligned}
U_{\epsilon}(f)=\left\{h \in \mathcal{F}: \sum_{|\alpha| \leq k}\left|D^{\alpha} f(x, \eta, \zeta)-D^{\alpha} h(x, \eta, \zeta)\right|<\epsilon(\eta, \zeta),\right. & \\
& \left.(x, \eta, \zeta) \in[0,1] \times \mathbb{R}^{2}\right\}
\end{aligned}
$$

(here $D^{\alpha}$ is the usual multi-index notation for partial derivatives); a subset $\mathcal{E} \subset \mathcal{F}$ is defined to be open if and only if for every $f \in \mathcal{E}$ there is a function $\epsilon$ such that the $\epsilon$-neighbourhood $U_{\epsilon}(f)$ of $f$ lies in $\mathcal{E}$. This topology is a slight modification of the usual Whitney topology as defined, for example, on p. 804 of [15]; the modification is simply to allow $f(\cdot, \eta, \zeta)$ to be a $C^{k}$ function on the closed set $[0,1]$ rather than on the open set $(0,1)$.

\section{Semi-trivial Solutions and SECONDARY Bifurcations}

In this section we consider the structure of the sets $\mathcal{C}_{m}^{1}, \mathcal{S}_{m}^{1}$, and discuss the local behaviour of the secondary bifurcations from these sets; by symmetry, similar results hold for the sets $\mathcal{C}_{n}^{2}, \mathcal{S}_{n}^{2}$, and the corresponding secondary bifurcations.

For any fixed $f \in \mathcal{F}$, we define a function $F: \mathbb{R} \times X_{1}^{0} \rightarrow Z$ by

$$
F(\mu, u)=L_{1} u-\mu u-f(\cdot, u, 0) u, \quad(\mu, u) \in \mathbb{R} \times X_{1}^{0} .
$$

The above assumptions ensure that $F$ is $C^{k}$. Furthermore, any point $(\mu, u, \nu, 0)$, $u \neq 0$, is a semi-trivial solution of (1)-(2) if and only if $F(\mu, u)=0$. Now, by adapting the methods and results in [12], it can be shown that there is a residual set $\mathcal{G}_{1}^{1} \subset \mathcal{F}$ such that if $f \in \mathcal{G}_{1}^{1}$ then the function $F$ is transverse, and hence for each $m \geq 0$ the set $\mathcal{C}_{m}^{1}$ is a $C^{k}$ curve in $\mathbb{R} \times X_{1}$ (the fact that the curve is $C^{k}$ at the point $\left(\mu_{m}^{0}, 0\right)$ follows from the form of the nonlinear term, $f(\cdot, u, 0) u$, and from the theory of bifurcation at a simple eigenvalue, see [7], rather than from transversality). Note that the results of [11] mentioned above showed that $\mathcal{C}_{m}^{1}$ is a continuum in the space $\mathbb{R} \times Y_{1}$. Thus $\mathcal{C}_{m}^{1}$ has a parametrization of the form

$$
\mathcal{C}_{m}^{1}=\left\{\left(\mu_{m}(s), u_{m}(s)\right): s \in \mathbb{R}\right\}
$$

where the functions $\mu_{m}: \mathbb{R} \rightarrow \mathbb{R}, u_{m}: \mathbb{R} \rightarrow X_{1}$ are $C^{k},\left(\mu_{m}(0), u_{m}(0)\right)=\left(\mu_{m}^{0}, 0\right)$, $u_{m}(s) \neq 0$ for $s \neq 0$, and $\left(\mu_{m, s}(s), u_{m, s}(s)\right) \neq(0,0)$ for all $s$ (the subscript $s$ here denotes differentiation with respect to $s)$. In addition, if $\mu_{m, s}\left(s^{0}\right)=0$ at some point $s^{0}$, then $\mu_{m, s s}\left(s^{0}\right) \neq 0$ (see [12]). Clearly, the corresponding set $\mathcal{S}_{m}^{1}$ is a 2-dimensional, $C^{k}$ manifold in $E$. 
We note at this point that various functions and sets defined in this paper depend on the functions $f$ and $g$ in the system (1)-(2). However, for simplicity of notation this dependence is not made explicit except where it is necessary to do so.

To discuss nodal properties of solutions we define the following sets: for any integer $r \geq 0$, let $S_{r}^{+}$(respectively, $S_{r}^{-}$) be the set of $w$ in $X_{1} \cup X_{2}$ such that $w$ has only simple zeros in $[0,1]$, has exactly $r$ such zeros in $(0,1)$, and $w(x)>0$ (respectively, $w(x)<0$ ) in some deleted neighbourhood of $x=0$. From now on $\sigma$ will denote an element of $\{+,-\}$. For each $\sigma$ let $T_{r}^{\sigma}=\mathbb{R} \times S_{r}^{\sigma}$. It follows from Rabinowitz' results that if the parametrization of $\mathcal{C}_{m}^{1}$ is chosen appropriately then, for $s \neq 0,\left(\mu_{m}(s), u_{m}(s)\right) \in T_{m}^{\sigma}$, where $\sigma=\operatorname{sgn}(s)$ (this definition of $\sigma$ has the natural interpretation). For each $\sigma$ we define the sets

$$
\mathcal{C}_{m}^{1, \sigma}=\left\{\left(\mu_{m}(s), u_{m}(s)\right): \sigma s>0\right\} \subset T_{m}^{\sigma}, \quad \mathcal{S}_{m}^{1, \sigma}=\mathcal{C}_{m}^{1, \sigma} \times \mathbb{R} \times\{0\} \subset E .
$$

Clearly, $\mathcal{C}_{m}^{1}=\mathcal{C}_{m}^{1,+} \cup \mathcal{C}_{m}^{1,-} \cup\left\{\left(\mu_{m}^{0}, 0\right)\right\}$, and the sets $\mathcal{S}_{m}^{1, \sigma}$ are sets of semi-trivial solutions of (1)-(2). We now consider the possibility of non-trivial solutions bifurcating from some of these semi-trivial solutions.

Suppose that $m \geq 0, f \in \mathcal{G}_{1}^{1}$, and that $\mathcal{C}_{m}^{1}$ has been parametrized as above. For a fixed point $\left(\mu_{m}(s), u_{m}(s)\right) \in \mathcal{C}_{m}^{1}, s \in \mathbb{R}$, linearizing equation (2) with respect to $v$ at $v=0$ yields the linear operator $L_{2, m}(s)-\nu I: X_{2} \rightarrow Z$, where $L_{2, m}(s)$ is the Sturm-Liouville operator defined by

$$
L_{2, m}(s) w=\left(L_{2}-g\left(\cdot, u_{m}(s), 0\right)\right) w, \quad w \in X_{2} .
$$

For each $s \in \mathbb{R}$ the operator $L_{2, m}(s)$ has a strictly increasing sequence of eigenvalues $\nu_{m n}(s), n \geq 0$, and corresponding eigenfunctions $\psi_{m n}(s)$. These eigenvalues are simple, in the sense of [7], so since $u_{m}(s)$ is a $C^{k}$ function of $s$, it can be shown that the functions $\nu_{m n}(s)$ are $C^{k}$ and the functions $\psi_{m n}(s)$ can be chosen so that they are $C^{k}$ and, for all $s \in \mathbb{R}, \psi_{m n}(s) \in S_{n}^{+}$and $\left\langle\psi_{m n}(s), \psi_{m n}(s)\right\rangle=1$ (where $\langle\cdot, \cdot \cdot\rangle$ denotes the usual $L^{2}(0,1)$ inner product). Also $\nu_{m n}(0)=\nu_{n}^{0}$, since $u_{m}(0)=0$.

Now define the set

$$
\mathcal{B}_{m n}^{1}=\left\{\left(\mu_{m}(s), u_{m}(s), \nu_{m n}(s), 0\right): s \in \mathbb{R}\right\} \subset \mathcal{S}_{m}^{1} \subset E,
$$

together with the corresponding subsets $\mathcal{B}_{m n}^{1, \sigma}$. The set $\mathcal{B}_{m n}^{1}$ is a smooth curve in $\mathcal{S}_{m}^{1}$ passing through the point $\left(\mu_{m}^{0}, 0, \nu_{n}^{0}, 0\right)$. We will show that, generically, secondary bifurcation to non-trivial solutions occurs at all points in $\mathcal{B}_{m n}^{1} \backslash\left\{\left(\mu_{m}^{0}, 0, \nu_{n}^{0}, 0\right)\right\}$, and that such bifurcations are 'simple'. Suppose that $(f, g) \in \mathcal{G}_{1}^{1} \times \mathcal{F}$. Choose a number $s^{1} \neq 0$, and let $b^{1}=\left(\mu_{m}\left(s^{1}\right), u_{m}\left(s^{1}\right), \nu_{m n}\left(s^{1}\right), 0\right) \in \mathcal{B}_{m n}^{1}$. Let $F_{(\mu, u)}^{1}$ denote the derivative of $F$ with respect to $(\mu, u)$ at the point $\left(\mu_{m}\left(s^{1}\right), u_{m}\left(s^{1}\right)\right)$, and let $P_{1}=N\left(F_{(\mu, u)}^{1}\right)^{\perp} \subset \mathbb{R} \times X_{1}, P_{2}=\mathbb{R} \times N\left(L_{2, m}\left(s^{1}\right)\right)^{\perp} \subset \mathbb{R} \times X_{2}$ (here and below, orthogonal subspaces in any of the spaces $X_{i}, Z$ are with respect to the inner product $\langle\cdot, \cdot\rangle$ or the natural inner product on any Cartesian product spaces). Let $\mathbb{R}_{0}=\mathbb{R} \backslash\{0\}$.

Lemma 2.1. There exist a ball $U \subset \mathbb{R}_{0} \times \mathbb{R}$, with centre $\left(s^{1}, 0\right)$, and $C^{k}$ functions $\bar{\mu}, \bar{u}, \bar{\nu}, \bar{v}$, defined on $U$ such that:

(i) for $(s, \alpha) \in U,(\bar{\mu}(s, \alpha), \bar{u}(s, \alpha)) \in P_{1},(\bar{\nu}(s, \alpha), \bar{v}(s, \alpha)) \in P_{2}$;

(ii) for each $(s, 0) \in U,(\bar{\mu}(s, 0), \bar{u}(s, 0), \bar{\nu}(s, 0), \bar{v}(s, 0))=(0,0,0,0)$;

(iii) for each $(s, \alpha) \in U$,

(6) $\left(\mu_{m}(s), u_{m}(s), \nu_{m n}(s), 0\right)+\left(\bar{\mu}(s, \alpha), \bar{u}(s, \alpha), \bar{\nu}(s, \alpha), \alpha\left(\psi_{m n}(s)+\bar{v}(s, \alpha)\right)\right)$,

is a solution of (1)-(2). 
Moreover, there is a neighbourhood $V \subset E$ of $b^{1}$ such that if $z=(\mu, u, \nu, v)$ is a solution of (1)-(2) in $V$, then either $z \in \mathcal{S}_{m}^{1}$ or there is $(s, \alpha) \in U$ such that $z$ is given by (6).

Proof. The problem is, in essence, one of bifurcation from simple eigenvalues (see [1] and [7]), so we will merely sketch a proof. We look for solutions of (1)-(2) having the form

$$
\left(\mu_{m}(s), u_{m}(s), \nu_{m n}(s), 0\right)+\left(\bar{\mu}, \bar{u}, \bar{\nu}, \alpha\left(\psi_{m n}(s)+\bar{v}\right)\right),
$$

with $(s, \alpha) \in \mathbb{R}^{2},(\bar{\mu}, \bar{u}) \in P_{1},(\bar{\nu}, \bar{v}) \in P_{2}$. Substituting this into (1)-(2) and dividing (2) by $\alpha$, we obtain the pair of equations

$$
\begin{gathered}
\left(L_{1}-\mu_{m}(s)-\bar{\mu}-f\left(\cdot, u_{m}(s)+\bar{u}, \alpha\left(\psi_{m n}(s)+\bar{v}\right)\right)\right)\left(u_{m}(s)+\bar{u}\right)=0, \\
\left(L_{2}-\nu_{m n}(s)-\bar{\nu}-g\left(\cdot, u_{m}(s)+\bar{u}, \alpha\left(\psi_{m n}(s)+\bar{v}\right)\right)\right)\left(\psi_{m n}(s)+\bar{v}\right)=0 .
\end{gathered}
$$

By construction, $(s, \alpha, \bar{\mu}, \bar{u}, \bar{\nu}, \bar{v})=(s, 0,0,0,0,0)$ satisfies these equations for all $s$. Now, the derivative with respect to $(\bar{\mu}, \bar{u}, \bar{\nu}, \bar{v})$ of the left hand sides of these equations at the point $(s, \alpha, \bar{\mu}, \bar{u}, \bar{\nu}, \bar{v})=\left(s^{1}, 0,0,0,0,0\right)$ is the operator

$$
(\widetilde{\mu}, \tilde{u}, \widetilde{\nu}, \tilde{v}) \rightarrow\left(\begin{array}{c}
F_{(\mu, u)}^{1}(\tilde{\mu}, \tilde{u}) \\
\left(L_{2, m}\left(s^{1}\right)-\nu_{m n}\left(s^{1}\right)\right) \tilde{v}-\widetilde{\nu} \psi_{m n}\left(s^{1}\right)
\end{array}\right) \in Z \times Z,
$$

where $(\widetilde{\mu}, \tilde{u}, \widetilde{\nu}, \tilde{v}) \in P_{1} \times P_{2}$. Since $F$ is transverse and $\nu_{m n}\left(s^{1}\right)$ is a simple eigenvalue of $L_{2, m}\left(s^{1}\right)$, this is an isomorphism from $P_{1} \times P_{2}$ to $Z \times Z$. Thus, by the implicit function theorem, the above pair of equations has a $C^{k}$ solution of the form $(\bar{\mu}(s, \alpha), \bar{u}(s, \alpha), \bar{\nu}(s, \alpha), \bar{v}(s, \alpha))$, defined for $(s, \alpha)$ near to $\left(s^{1}, 0\right)$, which satisfies $(\bar{\mu}(s, 0), \bar{u}(s, 0), \bar{\nu}(s, 0), \bar{v}(s, 0)) \equiv(0,0,0,0)$, for $s$ near 0 , and which corresponds to a solution of (1)-(2) having the desired form. The uniqueness assertions of the lemma are easily proved.

The result of Lemma 2.1 can be interpreted geometrically as follows. It is clear that putting $\alpha=0$ in (6) yields semi-trivial solutions lying on $\mathcal{B}_{m n}^{1}$, whereas $\alpha \neq 0$ yields non-trivial solutions (if $U$ is sufficiently small). Thus Lemma 2.1 shows that any semi-trivial solution $b^{1} \in \mathcal{B}_{m n}^{1}$ is a 'simple' bifurcation point, and near $b^{1}$ there is a smooth, connected, 2-dimensional manifold, $\mathcal{N}_{m n}^{1}\left(b^{1}\right)$ say, of solutions of (1)(2) which intersects the manifold $\mathcal{S}_{m}^{1}$ along the curve $\mathcal{B}_{m n}^{1}$ and is not tangential to $\mathcal{S}_{m}^{1}$. Any solution $z \in \mathcal{N}_{m n}^{1}\left(b^{1}\right) \backslash \mathcal{B}_{m n}^{1}$ is non-trivial. Solutions on $\mathcal{N}_{m n}^{1}\left(b^{1}\right)$ also have nodal properties. For any $\boldsymbol{\sigma}=\left(\sigma_{1}, \sigma_{2}\right) \in\{+,-\}^{2}$ let $T_{m n}^{\boldsymbol{\sigma}}=T_{m}^{\sigma_{1}} \times T_{n}^{\sigma_{2}} \subset E$. It follows from the properties of $\mathcal{B}_{m n}^{1}$ and $\psi_{m n}$ that if $(s, \alpha) \in U, \alpha \neq 0$, then the solution given by (6) lies in $T_{m n}^{\sigma}$, where $\boldsymbol{\sigma}=\left(\operatorname{sgn}\left(s^{1}\right), \operatorname{sgn}(\alpha)\right)$. Hence, putting $\sigma_{1}=\operatorname{sgn}\left(s^{1}\right)$, we may define, for each $\sigma_{2}$, the manifold $\mathcal{N}_{m n}^{1, \sigma_{2}}\left(b^{1}\right)=\mathcal{N}_{m n}^{1}\left(b^{1}\right) \cap T_{m n}^{\sigma}$, and we have

$$
\mathcal{N}_{m n}^{1}\left(b^{1}\right)=\mathcal{N}_{m n}^{1,+}\left(b^{1}\right) \cup \mathcal{N}_{m n}^{1,-}\left(b^{1}\right) \cup\left(\mathcal{B}_{m n}^{1} \cap V\right) .
$$

By symmetry, all the above constructions have analogues for semi-trivial solutions of the form $(\mu, 0, \nu, v), v \neq 0$, i.e., there exists a residual set $\mathcal{G}_{1}^{2} \subset \mathcal{F}$ such that if $(f, g) \in \mathcal{F} \times \mathcal{G}_{1}^{2}$ then for each $n \geq 0$, there is a curve of solutions $\mathcal{C}_{n}^{2} \subset \mathbb{R} \times X_{2}$ which bifurcates from $\left(\nu_{n}^{0}, 0\right)$, and a parametrization $\left(\nu_{n}(s), v_{n}(s)\right)$ of this curve; the corresponding set $\mathcal{S}_{n}^{2}$ is a 2-dimensional, $C^{k}$ manifold. Also, for each $m \geq 0$, 
there is a $C^{k}$ curve $\mathcal{B}_{m n}^{2}$ of secondary bifurcation points lying on $\mathcal{S}_{n}^{2}$. Note that the subscripts $m$ and $n$ here still refer to the nodal properties of the functions $u$ and $v$ respectively. Hence, all the above results hold for either type of semi-trivial solution when $(f, g)$ belongs to the residual set $\mathcal{G}_{2}:=\mathcal{G}_{1}^{1} \times \mathcal{G}_{1}^{2} \subset \mathcal{F}^{2}$.

Next, we consider the structure of the bifurcating sets near $\left(\mu_{m}^{0}, 0, \nu_{n}^{0}, 0\right)$. Let $\phi_{m}^{0} \in S_{m}^{+}$be the eigenfunction of $L_{1}$ corresponding to the eigenvalue $\mu_{m}^{0}$ with $\left\langle\phi_{m}^{0}, \phi_{m}^{0}\right\rangle=1$, and similarly let $\psi_{n}^{0} \in S_{n}^{+}$be the eigenfunction of $L_{2}$ corresponding to $\nu_{n}^{0}$; let $P_{1}=N\left(L_{1}-\mu_{m}^{0}\right)^{\perp} \subset X_{1}, P_{2}=N\left(L_{2}-\nu_{n}^{0}\right)^{\perp} \subset X_{2}$. Let

$$
\begin{array}{ll}
c_{m n, 11}=-\left\langle f_{u}(\cdot, 0,0)\left(\phi_{m}^{0}\right)^{2}, \phi_{m}^{0}\right\rangle, & c_{m n, 12}=-\left\langle f_{v}(\cdot, 0,0) \phi_{m}^{0} \psi_{n}^{0}, \phi_{m}^{0}\right\rangle, \\
c_{m n, 21}=-\left\langle g_{u}(\cdot, 0,0) \phi_{m}^{0} \psi_{n}^{0}, \psi_{n}^{0}\right\rangle, & c_{m n, 22}=-\left\langle g_{v}(\cdot, 0,0)\left(\psi_{n}^{0}\right)^{2}, \psi_{n}^{0}\right\rangle .
\end{array}
$$

We assume that the matrix $C_{m n}=\left(c_{m n, i j}\right)_{i, j=1}^{2}$ is non-singular. For $(f, g)$ in a suitable residual set $\mathcal{G}_{3} \subset \mathcal{G}_{2}$, this will be true for all $m, n \geq 0$.

Lemma 2.2. There exist a ball $U \subset \mathbb{R}^{2}$, with centre $(0,0)$, and $C^{k}$ functions $\bar{\mu}, \bar{u}$, $\bar{\nu}, \bar{v}$, defined on $U$ such that:

(i) for each $(\alpha, \beta) \in U: \bar{u}(\alpha, \beta) \in P_{1}, \bar{v}(\alpha, \beta) \in P_{2}$, and $\bar{\mu}(\alpha, \beta), \bar{\nu}(\alpha, \beta) \in \mathbb{R}$;

(ii) $\bar{u}(0,0)=0, \bar{v}(0,0)=0$ and $(\bar{\mu}(\alpha, \beta), \bar{\nu}(\alpha, \beta))=O\left(\alpha^{2}+\beta^{2}\right)$, as $(\alpha, \beta) \rightarrow$ $(0,0)$;

(iii) for each $(\alpha, \beta) \in U$, the formulae

$$
\begin{aligned}
& (\mu, u)=\left(\mu_{m}^{0}, 0\right)+\left(\alpha c_{m n, 11}+\beta c_{m n, 12}+\bar{\mu}(\alpha, \beta), \alpha\left(\phi_{m}^{0}+\bar{u}(\alpha, \beta)\right)\right), \\
& (\nu, v)=\left(\nu_{n}^{0}, 0\right)+\left(\alpha c_{m n, 21}+\beta c_{m n, 22}+\bar{\nu}(\alpha, \beta), \beta\left(\psi_{n}^{0}+\bar{v}(\alpha, \beta)\right)\right),
\end{aligned}
$$

yield a solution $(\mu, u, \nu, v)$ of (1)-(2).

Moreover, there is a neighbourhood $V \subset E$ of $\left(\mu_{m}^{0}, 0, \nu_{n}^{0}, 0\right)$ such that if $z=$ $(\mu, u, \nu, v)$ is a solution of (1)-(2) in $V$, then either $z \in \mathcal{S}_{m}^{1} \cup \mathcal{S}_{n}^{2}$ or there is $(\alpha, \beta) \in U$ such that $z$ is given by $(7)$.

Proof. The proof is similar to the proof of Lemma 2.1 - in this case the fact that the derivative operator is an isomorphism follows from the simplicity of both the eigenvalues $\mu_{m}^{0}, \nu_{n}^{0}$, rather than from a combination of transversality and simplicity, as previously. The details will be omitted.

Again there is a geometric interpretation of this result. In this case it is clear that putting $\alpha \neq 0, \beta=0$ (respectively $\alpha=0, \beta \neq 0$ ) in (7) yields semi-trivial solutions of (1)-(2) lying on $\mathcal{B}_{m n}^{1}$ (respectively $\mathcal{B}_{m n}^{2}$ ), while $\alpha \neq 0, \beta \neq 0$ yields non-trivial solutions (if $U$ is sufficiently small). Thus, near $\left(\mu_{m}^{0}, 0, \nu_{n}^{0}, 0\right)$ there is a smooth, connected, 2-dimensional manifold, $\mathcal{M}_{m n}$ say, of solutions of (1)-(2) which intersects the manifolds $\mathcal{S}_{m}^{1}, \mathcal{S}_{n}^{2}$, along the curves $\mathcal{B}_{m n}^{1}, \mathcal{B}_{m n}^{2}$. Any solution $z \in \mathcal{M}_{m n} \backslash\left(\mathcal{B}_{m n}^{1} \cup \mathcal{B}_{m n}^{2}\right)$ is non-trivial. Also, if $(\alpha, \beta) \in U, \alpha \beta \neq 0$, then the solution given by (7) lies in $T_{m n}^{\sigma}$, where $\sigma_{1}=\operatorname{sgn}(\alpha), \sigma_{2}=\operatorname{sgn}(\beta)$. Thus, letting $\mathcal{M}_{m n}^{\sigma}=\mathcal{M}_{m n} \cap T_{m n}^{\sigma}$ for each $\boldsymbol{\sigma} \in\{+,-\}^{2}$, we have

$$
\mathcal{M}_{m n}=\left(\bigcup_{\sigma} \mathcal{M}_{m n}^{\sigma}\right) \cup \mathcal{B}_{m n}^{1} \cup \mathcal{B}_{m n}^{2}
$$

The above lemmas have shown that bifurcation occurs at all points on $\mathcal{B}_{m n}^{i}$, $m, n \geq 0, i=1,2$. The method of proof of these lemmas can readily be used to show that bifurcation does not take place at any points on $\mathcal{S}_{m}^{1}, \mathcal{S}_{n}^{2}$, other than on the curves $\mathcal{B}_{m n}^{i}$. 


\section{NON-TRIVIAL SOLUTIONS}

Let $\mathcal{N}=\mathcal{N}(f, g)$ denote the set of non-trivial solutions of (1)-(2).

Theorem 3.1. There is a residual set $\mathcal{G}_{4} \subset \mathcal{F}^{2}$ such that if $(f, g) \in \mathcal{G}_{4}$, then the set $\mathcal{N}$ consists of a countable collection of 2-dimensional, $C^{k}$ manifolds.

Proof. Let $E_{0}=\mathbb{R} \times X_{1}^{0} \times \mathbb{R} \times X_{2}^{0} \subset E$ and, for any $(f, g) \in \mathcal{F}^{2}$, define a $C^{k}$ function $G(f, g): E_{0} \rightarrow Z \times Z$ by

$$
G(f, g)(\mu, u, \nu, v)=\left(\begin{array}{c}
L_{1} u-\mu u-f(\cdot, u, v) u \\
L_{2} v-\nu v-g(\cdot, u, v) v
\end{array}\right), \quad(\mu, u, \nu, v) \in E_{0} .
$$

Clearly, $\mathcal{N}=G(f, g)^{-1}(0)$. For any $\rho>1$, let

$$
A_{\rho}=\left\{(\mu, u, \nu, v) \in E_{0}:\|(\mu, u, \nu, v)\|<\rho,\|u\|_{2}>\rho^{-1},\|v\|_{2}>\rho^{-1}\right\},
$$

and let $\bar{A}_{\rho}$ be the closure of $A_{\rho}$. For any integer $r \geq 1$, let $\mathcal{T}_{r}$ be the set of functions $(f, g) \in \mathcal{F}^{2}$ such that the function $G(f, g)$ is transverse on $A_{r+\delta}$ for some sufficiently small $\delta>0$ (depending on $(f, g)$ ).

We first prove that each set $\mathcal{T}_{r}$ is open in $\mathcal{F}^{2}$. For any $(f, g) \in \mathcal{F}^{2}, \rho>1$, the set $\mathcal{N}(f, g) \cap \bar{A}_{\rho}$ is compact. Thus $(f, g) \notin \mathcal{T}_{r}$ if and only if there exists $z \in \mathcal{N}(f, g) \cap \bar{A}_{r}$ such that the operator $D G(f, g)(z)$ is not surjective (here the derivative is with respect to $(\mu, u, \nu, v))$. Thus, in deciding whether $(f, g) \in \mathcal{F}^{2}$, the values of $f$ and $g$ on $|u|>r,|v|>r$ are irrelevant. Therefore, it suffices to show that if $\left(f^{i}, g^{i}\right) \in \mathcal{F}^{2} \backslash \mathcal{T}_{r}, i=1,2, \ldots$, is a sequence converging to $\left(f^{0}, g^{0}\right) \in \mathcal{F}^{2}$ with respect to the $C^{k}$ topology on $\bar{\Omega} \times[-r, r]^{2}$, then $\left(f^{0}, g^{0}\right) \notin \mathcal{T}_{r}$. Now, for each $i$, there exists $z^{i} \in \mathcal{N}\left(f^{i}, g^{i}\right) \cap \bar{A}_{r}$ such that $D G\left(f^{i}, g^{i}\right)\left(z^{i}\right)$ is not surjective. Furthermore, it can be shown that the sequence $z^{i}$ converges (in $E$ ) to a point $z^{0}$ (using standard Sobolev embedding and regularity arguments; see for instance, the proof of Theorem 2.1 in [14] or the proof of Theorem 3.a.1 in [10]). Then, by continuity, $z^{0} \in \mathcal{N}\left(f^{0}, g^{0}\right) \cap \bar{A}_{r}$, and the operator $D G\left(f^{0}, g^{0}\right)\left(z^{0}\right)=\lim _{i \rightarrow \infty} D G\left(f^{i}, g^{i}\right)\left(z^{i}\right)$ cannot be surjective since this would contradict standard perturbation results for Fredholm operators (see for instance, Theorem 13.6 in [13]). Thus $\left(f^{0}, g^{0}\right) \notin \mathcal{T}_{r}$, which completes the proof that $\mathcal{T}_{r}$ is open in $\mathcal{F}^{2}$.

We will now show that the sets $\mathcal{T}_{r}$ are dense in $\mathcal{F}^{2}$. Choose an arbitrary, fixed $(f, g) \in \mathcal{F}^{2}$ and $r \geq 1$. Let $\theta_{r}: \mathbb{R} \rightarrow \mathbb{R}$ be a decreasing $C^{\infty}$ function such that $\theta_{r}(s)=1$ if $s \leq r+1$ and $\theta_{r}(s)=0$ if $s \geq r+2$. For any set of functions $\gamma=\left(\gamma_{i j}\right)_{i, j=1}^{2} \in \Gamma:=C^{k}([0,1])^{4}$ we define $f^{\gamma}:[0,1] \times \mathbb{R}^{2} \rightarrow \mathbb{R}$ by

$$
f^{\gamma}(x, \eta, \zeta)=f(x, \eta, \zeta)+\theta_{r}\left(\left(\eta^{2}+\zeta^{2}\right)^{1 / 2}\right)\left(\gamma_{11}(x) \eta+\gamma_{12}(x) \zeta\right),
$$

for $(x, \eta, \zeta) \in[0,1] \times \mathbb{R}^{2}$; we define $g^{\gamma}:[0,1] \times \mathbb{R}^{2} \rightarrow \mathbb{R}$ similarly, using $\gamma_{21}, \gamma_{22}$. Clearly, for any given positive functions $\epsilon_{1}, \epsilon_{2}$ (see the definition of the topology on $\mathcal{F}$ ) we have $f^{\gamma} \in U_{\epsilon_{1}}(f), g^{\gamma} \in U_{\epsilon_{2}}(g)$, for all sufficiently small $\gamma \in \Gamma$. We now define a function $H: A_{r+1} \times \Gamma \rightarrow Z \times Z$ by

$$
H(\mu, u, \nu, v, \gamma)=G\left(f^{\gamma}, g^{\gamma}\right)(\mu, u, \nu, v), \quad(\mu, u, \nu, v, \gamma) \in A_{r+1} \times \Gamma .
$$

Since $\Gamma$ is a Banach space (with norm $\|\gamma\|^{2}=\sum_{i, j=1}^{2}\left\|\gamma_{i j}\right\|_{k}^{2}$ ), we can differentiate $H$ with respect to $\gamma$ and apply transversality results. Clearly, $H$ is $C^{k}$.

Lemma 3.2. The derivative $D H\left(z^{0}\right)$ is surjective at any point $z^{0} \in H^{-1}(0)$. 
Proof. We must show that for any $\zeta=\left(\zeta_{1}, \zeta_{2}\right) \in Z \times Z$, the following equation can be solved for $(\widetilde{\mu}, \tilde{u}, \widetilde{\nu}, \tilde{v}, \widetilde{\gamma}) \in E \times \Gamma$ :

$$
H_{(u, v)}^{0}(\tilde{u}, \tilde{v})+H_{(\mu, \nu)}^{0}(\widetilde{\mu}, \widetilde{\nu})+H_{\gamma}^{0} \widetilde{\gamma}=\zeta,
$$

where $H_{(u, v)}^{0}, H_{(\mu, \nu)}^{0}, H_{\gamma}^{0}$, denote the derivatives at $z^{0}=\left(\mu^{0}, u^{0}, \nu^{0}, v^{0}, \gamma^{0}\right)$ with respect to $(u, v),(\mu, \nu)$ and $\gamma$ respectively; these operators have the form

$$
\begin{aligned}
& H_{(u, v)}^{0}(\tilde{u}, \tilde{v})=\left(\begin{array}{c}
\left(L_{1}-\mu^{0}-f^{0}\right) \tilde{u}-\left(f_{u}^{0} \tilde{u}+f_{v}^{0} \tilde{v}\right) u^{0} \\
\left(L_{2}-\nu^{0}-g^{0}\right) \tilde{v}-\left(g_{u}^{0} \tilde{u}+g_{v}^{0} \tilde{v}\right) v^{0}
\end{array}\right), \\
& H_{(\mu, \nu)}^{0}(\widetilde{\mu}, \widetilde{\nu})=\left(\begin{array}{c}
-\widetilde{\mu} u^{0} \\
-\widetilde{\nu} v^{0}
\end{array}\right), \quad H_{\gamma}^{0} \widetilde{\gamma}=\left(\begin{array}{c}
\widetilde{\gamma}_{11} u^{0} u^{0}+\widetilde{\gamma}_{12} u^{0} v^{0} \\
\widetilde{\gamma}_{21} u^{0} v^{0}+\widetilde{\gamma}_{22} v^{0} v^{0}
\end{array}\right)
\end{aligned}
$$

(where $f^{0}$ denotes $f^{\gamma^{0}}\left(\cdot, u^{0}, v^{0}\right)$, etc.). It can be verified that the operator $H_{(u, v)}^{0}$ is Fredholm and, if $\xi=\left(\xi_{1}, \xi_{2}\right) \in R\left(H_{(u, v)}^{0}\right)^{\perp} \subset Z \times Z$, then $\xi$ is a solution of a homogeneous coupled pair of linear ordinary differential equations. Let $d^{0}=$ $\operatorname{dim} R\left(H_{(u, v)}^{0}\right)^{\perp}$. If $d^{0}=0$ there is nothing further to prove, so suppose that $d^{0} \geq 1$ and let $\left\{\xi^{i} \in Z \times Z: i=1, \ldots, d^{0}\right\}$ be a basis for $R\left(H_{(u, v)}^{0}\right)^{\perp}$. Since the functions $u^{0}, v^{0}$ and $\xi^{i}, i=1, \ldots, d^{0}$, are all non-zero and can be regarded as solutions of homogeneous linear ordinary differential equations (or systems), it follows from the uniqueness of the solution of the initial value problem for such equations that these functions cannot be identically zero on any open set. Thus there must exist an open interval $I^{0} \subset(0,1)$ and a number $\delta>0$, such that for $x \in I^{0}$ we have $\left|u^{0}(x)\right|>\delta,\left|v^{0}(x)\right|>\delta$, and the functions $\xi^{i}, i=1, \ldots, d^{0}$, are linearly independent on $I^{0}$.

Now, since $u^{0}$ and $v^{0}$ are bounded away from 0 on $I^{0}$, for any $\psi=\left(\psi_{1}, \psi_{2}\right) \in$ $C_{0}^{\infty}\left(I^{0}\right)^{2}$, the equation $H_{\gamma}^{0} \widetilde{\gamma}=\psi$ has a solution $\widetilde{\gamma} \in C_{0}^{k}\left(I^{0}\right)^{4}$. Thus, we can choose $\gamma^{j} \in C_{0}^{k}\left(I^{0}\right)^{4}, j=1, \ldots, d^{0}$, such that $\left\langle H_{\gamma}^{0} \gamma^{j}, \xi^{i}\right\rangle=\delta_{i j}, i, j=1, \ldots, d^{0}\left(\delta_{i j}\right.$ is the Kronecker delta); thus the set $\left\{H_{\gamma}^{0} \gamma^{j}: j=1, \ldots, d^{0}\right\}$ spans a complement of $R\left(H_{(u, v)}^{0}\right)$ in $Z \times Z$, and so the operator $(\tilde{u}, \tilde{v}, \widetilde{\gamma}) \rightarrow H_{(u, v)}^{0}(\tilde{u}, \tilde{v})+H_{\gamma}^{0} \widetilde{\gamma} \in Z \times Z$ is surjective. This proves Lemma 3.2 .

It now follows from Lemma 3.2 and the transversality results in [14] (see Theorem 1.1 and Remark A.1 in [14] - it can readily be verified that $H$ satisfies the necessary Fredholm conditions) that there exists a residual set $\Theta \subset \Gamma$ such that if $\gamma \in \Theta$ then the mapping $H(\cdot, \gamma): A_{r+1} \rightarrow Z \times Z$, is transverse, i.e., $\left(f^{\gamma}, g^{\gamma}\right) \in \mathcal{T}_{r}$. Since $(f, g) \in \mathcal{F}^{2}$ was arbitrary, it follows from this that the set $\mathcal{T}_{r}$ is dense in $\mathcal{F}^{2}$. Now let $\mathcal{G}_{4}=\bigcap_{r=1}^{\infty} \mathcal{T}_{r}$. By construction, $\mathcal{G}_{4}$ is a residual subset of $\mathcal{F}^{2}$ and, for any $(f, g) \in \mathcal{G}_{4}$, the mapping $G(f, g): E_{0} \rightarrow Z \times Z$ is transverse. It follows that the zero set of this mapping is a 2-dimensional, $C^{k}$ manifold (see Theorem 73.G of [16]). Thus we have proved Theorem 3.1.

Theorem 3.1 shows that generically the set $\mathcal{N}$ is a countable collection of smooth manifolds. We now discuss how these manifolds link up with the local bifurcating manifolds constructed in Section 2. Let $\mathcal{G}_{5}=\mathcal{G}_{3} \cap \mathcal{G}_{4}$.

Theorem 3.3. Suppose that $(f, g) \in \mathcal{G}_{5}$. Then for each $m, n$, and $\boldsymbol{\sigma}=\left(\sigma_{1}, \sigma_{2}\right)$, there is a maximal connected $C^{k}$ manifold $\mathcal{N}_{m n}^{\sigma} \subset T_{m n}^{\sigma}$ of non-trivial solutions of (1)-(2) such that for each $i=1,2, \mathcal{B}_{m n}^{i, \sigma_{i}} \subset \overline{\mathcal{N}_{m n}^{\sigma}}$. The manifold $\mathcal{N}_{m n}:=\mathcal{B}_{m n}^{1} \cup \mathcal{B}_{m n}^{2} \cup$ $\left(\bigcup_{\sigma} \mathcal{N}_{m n}^{\sigma}\right)$ is also a connected $C^{k}$ manifold. Near any point $b \in \mathcal{B}_{m n}^{i}$, the manifold 
$\mathcal{N}_{m n}$ coincides with the corresponding local manifold constructed in Lemma 2.1 or Lemma 2.2.

Proof. For each $m, n, \boldsymbol{\sigma}$, the union of the local manifolds from Lemmas 2.1 and 2.2 ,

$$
\bigcup_{b^{1} \in \mathcal{B}_{m n}^{1, \sigma_{1}}} \mathcal{N}_{m n}^{1, \sigma_{1}}\left(b^{1}\right) \cup \bigcup_{b^{2} \in \mathcal{B}_{m n}^{2, \sigma_{2}}} \mathcal{N}_{m n}^{2, \sigma_{2}}\left(b^{2}\right) \cup \mathcal{M}_{m n}^{\sigma}
$$

is a connected $C^{k}$ manifold of non-trivial solutions of (1)-(2) (the manifold $\mathcal{M}_{m n}^{\sigma}$ connects the contributions from the points $b^{1} \in \mathcal{B}_{m n}^{1, \sigma_{1}}$ and $\left.b^{2} \in \mathcal{B}_{m n}^{2, \sigma_{2}}\right)$. Let $\mathcal{N}_{m n}^{\sigma}$ be the connected component of $\mathcal{N}$ containing this union. The nodal properties of the solutions cannot change along a component of $\mathcal{N}$, so $\mathcal{N}_{m n}^{\sigma} \subset T_{m n}^{\sigma}$ (since all the local manifolds lie in $T_{m n}^{\sigma}$ ). By Theorem $3.1, \mathcal{N}_{m n}^{\sigma}$ is a $C^{k}$ manifold. It follows from Lemmas 2.1 and 2.2 that $\mathcal{N}_{m n}$ is also $C^{k}$.

Theorem 3.3 shows that for each $\boldsymbol{\sigma}$ the curves $\mathcal{B}_{m n}^{1, \sigma_{1}}, \mathcal{B}_{m n}^{2, \sigma_{2}}$ are 'linked' by the manifold $\mathcal{N}_{m n}^{\sigma}$ (in fact, every pair of points on the two curves can be connected by a curve lying in $\mathcal{N}_{m n}^{\sigma}$ ).

\section{A PRIORI INFORMATION AND GLOBAL BEHAVIOUR}

Throughout this section we suppose that $(f, g) \in \mathcal{G}_{5}$. For any given $m$ and $n$ it is possible that the manifold $\mathcal{N}_{m n}$ of Theorem 3.3 could meet semi-trivial solutions other than those on the curves $\mathcal{B}_{m n}^{i}$ (corresponding to solutions of (3) or (4) which do not lie on $\mathcal{C}_{m}^{1}$ or $\left.\mathcal{C}_{n}^{2}\right)$. However, we can extend $\mathcal{N}_{m n}$ 'through' such solutions as follows. Since $(f, g) \in \mathcal{G}_{2}$, an analogue of Lemma 2.1 holds near any semi-trivial solution $z \in \overline{\mathcal{N}}$ (not just those on $\mathcal{B}_{m n}^{i}$ ), and shows that, locally, $\overline{\mathcal{N}}$ is a connected $C^{k}$ manifold crossing the set of semi-trivial solutions near $z$ along a smooth curve. The number of nodal zeros of $u$ and $v$ is preserved in such a crossing, although the sign of either $u$ or $v$ near $x=0$ will change. Let $\mathcal{E}_{m n}$ be the component of $\overline{\mathcal{N}}$ which contains $\mathcal{N}_{m n}$. The above observations show that $\mathcal{E}_{m n}$ is a connected $C^{k}$ manifold and $\mathcal{E}_{m n} \cap \mathcal{N} \subset T_{m n}:=\bigcup_{\boldsymbol{\sigma}} T_{m n}^{\boldsymbol{\sigma}}$.

The manifold $\mathcal{E}_{m n}$ has certain global properties. For any $\mu \in \mathbb{R}$, let $E^{1}(\mu)=$ $\{\mu\} \times X_{1} \times \mathbb{R} \times X_{2}$ and let $\mathcal{E}_{m n}^{1}(\mu)=\mathcal{E}_{m n} \cap E^{1}(\mu)$ (we can also define $\mathcal{E}_{m n}^{2}(\nu)$ for $\nu \in \mathbb{R}$ in a similar manner, and similar results hold for these sets). As noted in Section $2, \mu_{m, s s}(s) \neq 0$ whenever $\mu_{m, s}(s)=0$, so the set of $s$ for which $\mu_{m, s}(s)=0$ is discrete. Now choose $s^{1} \neq 0$ such that $\mu_{m, s}\left(s^{1}\right) \neq 0$, and let $\mu^{1}=\mu_{m}\left(s^{1}\right)$, $b^{1}=\left(\mu^{1}, u_{m}\left(s^{1}\right), \nu_{m n}\left(s^{1}\right), 0\right)$. By the results of Lemma 2.1 there is a neighbourhood $W \subset E^{1}\left(\mu^{1}\right)$ of $b^{1}$ such that $\mathcal{E}_{m n}^{1}\left(\mu^{1}\right) \cap W$ is a $C^{k}$ curve. A similar result holds near any semi-trivial solution $z \in \mathcal{E}_{m n}^{1}\left(\mu^{1}\right)$, even if $z \notin \mathcal{B}_{m n}^{i}$ (for suitable $s^{1}$ ). Next, consider the non-trivial part $\mathcal{E}_{m n}^{1}(\mu) \cap \mathcal{N}$ of $\mathcal{E}_{m n}^{1}(\mu)$. By examining the proof of Theorem 3 it can be seen that the $\mu$ derivative of $H$ was not used, so a similar result holds if $\mu$ is regarded as fixed. Thus there is a residual set $\mathcal{G}_{6}(\mu) \subset \mathcal{F}^{2}$ such that if $(f, g) \in \mathcal{G}_{6}(\mu)$ then $\mathcal{E}_{m n}^{1}(\mu) \cap \mathcal{N}$ is a collection (possibly empty) of $C^{k}$ curves. Thus, with some more work, we can construct residual sets $\mathcal{G}_{7} \subset \mathcal{G}_{5}$ and $\mathcal{H} \subset \mathbb{R}$ such that if $(f, g) \in \mathcal{G}_{7}$ and $\mu \in \mathcal{H}$, then the complete set $\mathcal{E}_{m n}^{1}(\mu)$ is a collection of $C^{k}$ curves (this collection may be empty, but is certainly non-empty if $\mu=\mu_{m}(s)$ for some $s \neq 0$ ). It also follows (from transversality) that the individual curves in $\mathcal{E}_{m n}^{1}(\mu)$ must either be closed loops or must be unbounded (at each end). 
In many problems a priori bounds on the size of the solutions or the numbers of solutions of the system are available. We will consider the implications of the following two assumptions, which hold for many systems.

A1. For all $m, n, \sigma$, any solution $(\mu, u) \in T_{m}^{\sigma}$ of (3) (respectively $(\nu, v) \in T_{n}^{\sigma}$ of (4)) belongs to $\mathcal{C}_{m}^{1, \sigma}$ (respectively $\mathcal{C}_{n}^{2, \sigma}$ ).

A2. For all $m, n$, there exist constants $\mu_{m n}^{*}, \nu_{m n}^{*}$, and a continuous function $\beta_{m n}:[0, \infty) \rightarrow[0, \infty)$ such that if $(\mu, u, \nu, v) \in T_{m n}$ is a solution of (1)-(2), then $\mu \geq \mu_{m n}^{*}, \nu \geq \nu_{m n}^{*}$ and $\|u\|_{2}+\|v\|_{2} \leq \beta_{m n}(|\mu|+|\nu|)$.

Assumption A1 is a generalization of Hypothesis $\mathrm{H} 2$ in [5]; see, for instance, the references in [5] and in [2] for conditions on $f$ and $g$ which ensure that A1 holds. Conditions which ensure that A2 holds are discussed in [5], and elsewhere.

If A1 holds then $\mathcal{N}_{m n}$ meets no semi-trivial solutions other than those on the curves $\mathcal{B}_{m n}^{i}$, so $\mathcal{E}_{m n}=\mathcal{N}_{m n}$. Also, if $(f, g) \in \mathcal{G}_{7}, \mu \in \mathcal{H}, \mu \neq \mu_{m}^{0}$, and if a curve in $\mathcal{E}_{m n}(\mu)$ forms a loop, then by nodal properties this loop must either contain no semitrivial solution or must intersect, successively, the curves $\mathcal{B}_{m n}^{1,+}, \mathcal{B}_{m n}^{2,+}, \mathcal{B}_{m n}^{1,-}, \mathcal{B}_{m n}^{2,-}$, and the nodal structure of the solutions on the loop changes at each intersection.

For each $i$, let $\mathcal{P}_{m n}^{i}$ denote the projection of $\mathcal{B}_{m n}^{i}$ onto the $\mu \nu$-plane. If $\mathrm{A} 2$ holds then, since $\mathcal{C}_{m}^{i}$ is unbounded in $\mathbb{R} \times Y_{i}$, the projection $\mathcal{P}_{m n}^{i}$ must be unbounded. Furthermore, if $(f, g) \in \mathcal{G}_{7}$ and $\mu \in \mathcal{H}$, then either the set $\mathcal{E}_{m n}^{1}(\mu)$ is a loop or its projection onto the $\mu \nu$-plane is unbounded in the positive $\nu$ direction. In particular, slightly more precise a priori bounds on the solutions in the continua $\mathcal{C}_{m}^{1}, \mathcal{C}_{n}^{2}$ ensure that there will be an unbounded region $Q$ in the $\mu \nu$-plane above $\mathcal{P}_{m n}^{1}$ and to the right of $\mathcal{P}_{m n}^{2}$. It then follows from the above results that for each $\boldsymbol{\sigma}$, the set $Q$ will be completely covered by the projection of $\mathcal{N}_{m n}^{\sigma}$ onto the $\mu \nu$-plane, i.e., for any $(\mu, \nu) \in Q$ there will be at least one corresponding non-trivial solution $(\mu, u, \nu, v) \in \mathcal{N}_{m n}^{\sigma}$. A similar result is proved in Theorem 4.1 in [5].

Remark 4.1. All the above results hold if $f$ and $g$ depend on $u^{\prime}$ and $v^{\prime}$, after making some minor changes (for instance, to the definition of $\mathcal{F}$ ). Also, most of the results can be extended, by an inductive process, to systems having a similar form but with more equations. For instance, with three equations in three variables, say $u, v, w$, we consider non-trivial solutions having all three variables non-zero and semi-trivial solutions having either one or two of these variables identically zero. Such semi-trivial solutions correspond to non-trivial or semi-trivial solutions of systems of two equations, so the above results describe the structure of the set of semi-trivial solutions of the three equation system while, generically, the set of nontrivial solutions can be shown to be a collection of smooth, 3-dimensional manifolds. The linking between the semi-trivial and non-trivial solution sets of this system can also be described generically.

\section{REFERENCES}

[1] J. C. Alexander And S. S. Antman, Global and local behaviour of bifurcating multidimensional continua of solutions for multiparameter nonlinear eigenvalue problems, Arch. Rat. Mech. Anal. 76 (1981), 339-354. MR 82k:58030

[2] J. Blat and K. J. Brown, Bifurcation of steady-state solutions in predator-prey and competition systems, Proc. Roy. Soc. Edin. 97A (1984), 21-34. MR 85k:92053

[3] R. S. Cantrell, Global higher bifurcations in coupled systems of nonlinear eigenvalue problems, Proc. Roy. Soc. Edin. 106A (1987), 113-120. MR 88h:92035

[4] R. S. Cantrell, Global preservation of nodal structure in coupled systems of nonlinear Sturm-Liouville boundary value problems, Proc. Amer. Math. Soc. 107 (1989), 633-644. MR 90i: 34037 
[5] R. S. Cantrell, Parameter ranges for the existence of solutions whose state components have specified nodal structure in coupled multiparameter systems of nonlinear Sturm-Liouville boundary value problems, Proc. Roy. Soc. Edin. 119A (1991), 347-365. MR 93d:34031

[6] R. S. Cantrell and C. Cosner, On the steady-state problem for the Volterra-Lotka competition model with diffusion, Houston J. Math. 13 (1987), 337-352. MR 89d:92052

[7] M. G. Crandall and P. H. Rabinowitz, Bifurcation from simple eigenvalues, J. Funct. Anal. 8 (1971), 321-340. MR 44:5836

[8] E. N. DANCER, On the structure of solutions of non-linear eigenvalue problems, Indiana Univ. Math. J. 23 (1974), 1069-1076. MR 50:1065

[9] D. Gilbarg and N. S. Trudinger, Elliptic Partial Differential Equations of Second Order, Springer, 1983. MR 86c:35035

[10] P. Brunovsky and P. Polacik, The Morse-Smale structure of a generic reaction-diffusion equation in higher space dimension, J. Diff. Equns. 135 (1997), 129-181. CMP 97:08

[11] P. H. Rabinowitz, Some global results for nonlinear eigenvalue problems, J. Funct. Anal. 7 (1971), 487-513. MR 46:745

[12] B. P. Rynne, Genericity of hyperbolicity and saddle-node bifurcations in reaction-diffusion equations depending on a parameter, J. Appl. Math. Phys. (ZAMP) 47 (1996), 730-739. MR 97h:35105

[13] A. E. Taylor And D. C. Lay, Introduction to Functional Analysis, Second Edition, Wiley, 1980. MR 81b:46001

[14] J. C. Saut and R. Temam, Generic properties of nonlinear boundary value problems, Comm. in PDE 4 (1979), 293-319. MR 80i:35080

[15] E. Zeidler, Nonlinear Functional Analysis and its Applications, Vol I, Springer, 1986. MR 87f: 47083

[16] E. ZeIDLER, Nonlinear Functional Analysis and its Applications, Vol IV, Springer, 1988. MR 90b:00004

Department of Mathematics, Heriot-Watt University, Riccarton, Edinburgh EH14 4AS, SCOTLAND

E-mail address: bryan@ma.hw.ac.uk 